\title{
tic\&société
}

Vol. 6, $\mathrm{N}^{\circ} 1$ | Second semestre 2012

Mutations du journalisme : nouveaux dispositifs, nouvelles pratiques

\section{La certification de contenus collaboratifs à l'agence photo Citizenside :}

entre procédures nouvelles et fondamentaux anciens

Jérémie Nicey

\section{OpenEdition \\ Journals}

Édition électronique

URL : http://journals.openedition.org/ticetsociete/1183

DOI : 10.4000/ticetsociete. 1183

Éditeur

Association ARTIC

Référence électronique

Jérémie Nicey, « La certification de contenus collaboratifs à l'agence photo Citizenside : », tic\&société [En ligne], Vol. 6, $\mathrm{N}^{\circ} 1$ I Second semestre 2012, mis en ligne le 28 novembre 2012, consulté le 20 avril 2019. URL : http://journals.openedition.org/ticetsociete/1183 ; DOI : 10.4000/ticetsociete.1183 


\title{
La certification de contenus collaboratifs à l'agence photo Citizenside : entre procédures nouvelles et fondamentaux anciens
}

\author{
Jérémie NICEY \\ Laboratoire CIM : Communication Information Médias \\ Université Sorbonne Nouvelle - Paris 3 \\ 1 , rue Censier \\ 75005 Paris \\ France
}

jeremie.nicey@univ-paris3.fr / jeremie.nicey@univ-tours.fr

Jérémie NICEY, docteur en Sciences de l'information et de la communication, a achevé fin 2011 un post-doctorat sur les archives de l'Agence France-Presse, au laboratoire CIM de I'Université Sorbonne Nouvelle Paris 3. Après avoir enseigné dans plusieurs universités et écoles de journalisme de la région parisienne, il intègre en 2012 l'Ecole publique de journalisme de Tours (rattachée à l'Université François-Rabelais de Tours) en tant que Maître de conférences. 


\title{
La certification de contenus collaboratifs à l'agence photo Citizenside : entre procédures nouvelles et fondamentaux anciens
}

\begin{abstract}
Résumé
La pertinence du journalisme participatif - ou collaboratif - est analysée et interrogée ici par l'auteur à travers l'étude de cas de Citizenside, jeune agence photo professionnelle dont l'Agence France-Presse détient 34\%, qui collecte en ligne les images de contributeurs et les revend pour eux aux médias mainstream. L'enjeu principal porte sur la vérification des contenus reçus : au-delà des pratiques nouvelles, des procédures et outils nouveaux (métadonnées, géolocalisation et community management), il révèle, au fond, l'application de fondamentaux anciens du journalisme, à savoir le croisement des sources, l'investigation approfondie et la mise en attente de la publication en cas de doute. L'intérêt du modèle va même plus loin, avec l'amélioration des compétences des contributeurs. Dès lors, ceux-ci ne peuvent plus être considérés comme de véritables "amateurs". La collaboration des différents acteurs de ce nouveau circuit d'information profite ainsi à chacun d'entre eux. La dimension de ce nouveau dispositif média, pour l'instant confidentielle, est-elle amenée à s'élargir? Et quels défis cela pose-t-il ?
\end{abstract}

Mots-clés : journalisme participatif, journalisme citoyen, UGC (usergenerated content), Pro-Am, amateurs, AFP.

\section{Abstract}

The relevance of participatory - or collaborative - journalism is analysed and questioned by the author, through the case study of Citizenside, a young professional photo agency in which Agence France-Presse holds a 34\% stake. This agency collects the images of online contributors and helps sell them to mainstream media for a percentage. The main challenge is to check 
and double-check the content. Beyond new practices, new procedures and tools (metadata, geolocation and community management), observation reveals the application of fundamental basics of journalism, which are ancient: the use of different sources, indepth investigation and delayed publishing in case of dubious images. The model is also of interest because of the progress of the contributors' skills. Thus, they can no longer be called 'amateurs'. The collaboration of the different actors in this new circuit of news therefore benefits all of them. Will this new media trend, still rather small, expand? What challenges would such growth raise?

Keywords: participatory journalism, citizen journalism, UGC (user-generated content), Pro-Am, amateurs, AFP.

\section{Resumen}

La pertinencia del periodismo participativo -o colaborativo- es analizada y cuestionada aquí por el autor a través del estudio del caso de Citizenside, joven agencia de fotografía profesional -en la cual la agencia France-Presse tiene una participación del 34\%-. Esta joven agencia se dedica a la colecta de imágenes, propiedad de los contribuyentes, que después las vende a los medios mainstream. El reto principal se concentra en la verificación de los contenidos recibidos. $Y$, más que de prácticas nuevas se trata de nuevos procedimientos y de nuevos instrumentos (metadatos, geolocalización y community management); con ello se pone en evidencia la aplicación de las bases del periodismo clásico: el cruce de fuentes, la investigación en profundidad y la colocación en la fila de espera de la publicación, en caso de duda. El interés del modelo que se presenta va más allá y muestra el perfeccionamiento de las competencias de los contribuyentes pues éstos no pueden ser considerados como verdaderos "amateurs". La colaboración de los diversos actores en este nuevo circuito de información favorece así a todos y a cada uno de ellos. La dimensión de este nuevo dispositivo mediático, por el momento confidencial, ¿está destinada a prolongarse? ¿Cuáles son los desafíos que ello implica?

Palabras clave: periodismo participativo, periodismo ciudadano, UGC (user-generated content), Pro-Am, amateurs, AFP. 
La certification de contenus collaboratifs à l'agence photo Citizenside Entre procédures nouvelles et fondamentaux anciens

Si mutation de la production de contenus informationnels il y a, celle-ci passe notamment par la place prise par les non professionnels. Après le premier temps des blogs à la fin des années 1990 et au début des années 2000, plusieurs éléments se sont révélés favorables à la participation croissante des citoyens dans les dispositifs médiatiques : les progrès techniques (équipements, connexions et débits), l'émergence de sites d'autopublication (regroupés sous le modèle web 2.0) et la baisse des coûts des appareils de captures photo et vidéo (appareils numériques puis smartphones) ont conduit à la mise à disposition d'images dites « amateurs » auprès des rédactions journalistiques, et à leur intégration progressive et désormais récurrente dans la fabrication des récits d'information.

Dans ce contexte, les fournisseurs traditionnels d'information, en premier lieu les agences de presse, telle l'Agence France-Presse (AFP) en France, voient leur production concurrencée (Boyd-Barrett, 2010 ; Palmer, 2011) par des acteurs en ligne, pour une part non professionnelle. En parallèle, certaines nouvelles agences spécialisées - qui sur une aire géographique, qui sur un support, photo et vidéo par exemple - se sont constituées précisément en fondant leur modèle sur le « journalisme participatif » ou « journalisme citoyen » (Lasica, 2003 ; Paulussen et al., 2007 ; Allan et al., 2009 ; Singer et al., 2011). Elles collectent les contenus dits «amateurs" puis les proposent - contre rétribution - aux médias mainstream. Avec un défi : fiabiliser leur offre de contenus (Rebillard, 2007 ; Bouquillion et Matthews, 2010).

Cet article se propose d'analyser le fonctionnement et le modèle de Citizenside, agence photo de type web 2.0, née en France en 2006. Trois étapes méthodologiques sont au fondement des pistes de réflexion qui vont suivre: 1/ une recherche postdoctorale à l'Agence France-Presse (projet «Archives-AFP » conduit par le laboratoire CIM, Université Sorbonne nouvelle Paris 3, soutenu par l'Agence nationale de la recherche), permettant de saisir les mutations et les liens entre acteurs traditionnels et nouveaux dans la collecte des images, incluant à la fois la dimension du contenu et celle de sa diffusion (donc de la clientèle médias) ; 2 / une approche initiale de l'agence Citizenside (processus en cours d'approfondissement) à la fois par trois entretiens semidirectifs (d'une durée approximative d'une heure, avec un des fondateurs de l'agence, le rédacteur en chef et son adjoint) et par une séance d'observation de type ethnographique, pour cerner le modus operandi de l'agence, autrement dit les stratégies d'origine et à venir définies par ses dirigeants, ainsi que les 
Jérémie NICEY

dispositifs techniques et les pratiques de sa rédaction ; 3 / un repérage attentif des recherches portant sur la dimension participative du journalisme actuel, de façon à en vérifier la validité concernant le cas ici étudié.

Certains événements nationaux voire internationaux ont, entre 2009 et 2011, en quelque sorte crédibilisé Citizenside au sein du paysage professionnel. Pour autant, nous chercherons ici à plonger sous la partie émergée que constituent les images publiées et le site web de l'agence. Quelles sont les pratiques de collecte et de fiabilisation des images des citoyens contributeurs ? Sur quoi sont-elles fondées et quels en sont les enjeux ? Et ces pratiques rejoignent-elles celles des confrères exerçant dans les rédactions dites traditionnelles, y compris pour leurs versions en ligne? Ce triple questionnement va nous permettre d'une part d'examiner les ressorts du journalisme participatif et de la collaboration entre Citizenside et l'AFP, d'autre part de scruter la validité de la certification des contenus collaboratifs (dits "amateurs"), et in fine d'interroger les critères de qualité - nouveaux et anciens - du journalisme.

\title{
1. Une « double » collaboration : journalisme participatif et « partenariat » AFP-Citizenside
}

\author{
1.1 Participation des usagers des médias : de " UGC » à "Pro-Am "
}

Dans la rédaction de Citizenside (à Paris) est affichée une maxime du journaliste et critique de presse états-unien Jay Rosen: "Professional journalism has been optimized for low participation» (Rosen, 2011). Le journalisme participatif semble précisément avoir pour ambition de renverser une telle logique, et de redéfinir les rapports entre les publics et les appareils productifs médiatiques. Si la participation des amateurs dans la production informationnelle n'est pas à proprement parler une nouveauté (images de l'assassinat de JFK en 1963, pouvoir de la radio via la parole citoyenne dans les années 1960 et 1970, etc.), elle s'est considérablement accrue depuis l'ère numérique. Davantage que le 11 septembre 2001, c'est sans doute l'année 2004 qui constitue en France une charnière dans ce domaine: d'une part, l'avènement de l'Internet à haut débit - qui augmente considérablement la vitesse de publication, de circulation et de consultation des contenus - et, d'autre part, l'utilisation massive de vidéos " amateurs » lors du tsunami en Asie du Sud-est (Nicey, 2008) ont contribué à un intérêt réel vis-à-vis des capacités de production par les publics. Ces capacités entrent d'ailleurs en résonance avec le besoin d'« autonomisation ", notion plus connue sous son appellation anglaise « empowerment » (Jenkins, 2006). 
La certification de contenus collaboratifs à l'agence photo Citizenside Entre procédures nouvelles et fondamentaux anciens

La plus-value de ce phénomène et son amplification avec Internet résident dans l'immédiateté et l'interactivité (Paterson et Domingo, 2008), auxquelles s'ajoute un critère essentiel, l'authenticité. C'est sur ce principe et dans cette période charnière que furent lancés plusieurs sites web destinés à collecter la matière produite par les usagers (ou "UGC » pour «User Generated Content "), matière en l'occurrence ici informationnelle : OhmyNews (Corée du Sud, lancé en 2000) ; NowPublic (États-Unis, 2005) ; iReport (États-Unis, lancé par CNN en 2006) ; Citizenside (France, 2006) ; Demotix (Royaume-Uni, 2008) ; etc. Avant de se développer à l'international et de gagner en reconnaissance, ces plateformes firent leurs premiers pas avec un objectif principal : placer les contenus de leurs contributeurs auprès des médias classiques, et en tirer un bénéfice financier, qu'elles partagent pour la plupart avec les auteurs desdits contenus (cf. infra). Le recours croissant à des contenus produits par les publics (ou crowdsourcing) et l'attention portée par les médias installés à soigner leur propre communauté de lecteurs-usagers (ou «produsage", selon Axel Bruns, 2010) a même conduit à une appellation spécifique, voire un modèle: les contenus «Pro-Am », autrement dit les contenus issus de la collaboration entre professionnels et amateurs.

\subsection{Structuration de l'offre et « business to business »}

Citizenside entre pleinement dans ce nouveau paradigme Pro-Am. Né sous le nom de "Scooplive ", le site fut lancé en juin 2006 - en ce sens, l'entreprise n'est désormais plus une start-up à proprement parler. Le concept fut imaginé un an plus tôt, à la suite des attentats à la bombe à Londres le 7 juillet 2005, par Matthieu Stefani. Ce dernier, alors sur place, constata plusieurs biais dans l'utilisation des contenus envoyés aux télévisions par les citoyens (plus réactifs, car présents au moment de l'événement et en raison du périmètre de sécurité contraignant pour les journalistes); ces biais étaient de différentes natures: manque de qualité des images, fiabilité non garantie, retards dans la collecte et la diffusion des images (en particulier concernant les alertes de la BBC). Stefani perçut les défis ainsi posés au journalisme. De retour à Paris, il réunit deux de ses jeunes amis entrepreneurs (Philippe Checinski et Julien Robert) pour lancer une plateforme en ligne pouvant structurer l'offre de contenus amateurs à destination des professionnels des médias, c'est-à-dire un ancrage «B-2-B » ("business-to-business ») en terme de production journalistique ; on comprend dès lors en quoi l'agence elle-même n'est pas "amateur ". La philosophie de Citizenside - nouveaux types de contenus, nouveaux angles, nouvelles approches de l'information - repose pourtant sur ses contributeurs, qualifiés en interne de « membres » (nous soulignerons plus loin cet aspect communautaire) 
Jérémie NICEY

ou de "témoins d'actualité ". Ces contributeurs - après contrôle et vérification par la rédaction, cf. infra - sont à l'origine de 300 à 500 photographies publiées chaque jour sur le site (environ 1000 les jours de manifestations massives à l'appel des syndicats) ; les " citoyens participants " conservent leurs droits d'auteur et tirent entre $50 \%$ et $65 \%$ de la vente de ces images aux médias (pour les plus communes, de 10 euros pour un site web peu visité à 630 euros pour une couverture de magazine de presse écrite). L'agence a par ailleurs intégré à ses services le système de «marques blanches" (service commercialisé chez Citizenside sous le nom de Kit Reporter) qu'affectionnent certains clients-médias: tout en restant invisible à l'usager, l'agence photo travaille pour eux en organisant la plateforme de collecte de contenus auprès de leurs propres communautés. C'est le cas du Parisien, de Metro et 20 Minutes, mais aussi, entre autres, de la radio RTL et de la chaîne de télévision d'information en continu BFM-TV; plus récemment, fin 2011, le groupe britannique de presse régionale Archant a acquis ces mêmes services. Cet aspect communautaire s'avère décisif : c'est une des raisons qui a poussé l'agence à choisir comme slogan «The Global news community », ce qui traduit par ailleurs une opérabilité dans environ 150 pays - bien que la majorité des contributeurs reste européenne et même française.

\subsection{L'AFP et Citizenside : réciprocité d'intérêts}

L'agence photo Citizenside a établi un autre niveau de collaboration, à caractère encore plus stratégique vis-à-vis des pairs. Dès novembre 2007, l'une des trois plus puissantes - et la plus ancienne - agences mondiales d'information (Palmer et Boyd-Barrett, 1981), l'Agence France-Presse, a conclu une prise de participation à $34 \%$ dans la start-up - c'est d'ailleurs à cette occasion que cette dernière, abandonnant le nom Scooplive, fut renommée Citizenside. Dans le contexte de concurrence globale et accrue sur Internet, l'AFP tente en effet de se positionner, notamment par la mise en avant du droit d'auteur et la lutte contre la copie de ses contenus, par exemple en ayant poursuivi Google Actualités en 2005, lui réclamant 17,5 millions de dollars de dédommagements (avant de finalement signer un accord avec le portail américain en 2007). Pour autant, l'AFP cherche à ne pas occulter les réseaux en ligne (Palmer et Nicey, 2011) - elle a ainsi en 2011 défini et rendu publiques ses propres règles d'utilisation de Twitter et de Facebook, suivant ainsi les mutations imposées par le web social (Millerand, Proulx et Rueff, 2010).

Qu'a l'AFP à gagner d'une collaboration avec une agence de contenus dits " amateurs "? Premier élément de réponse : l'intérêt d'avoir un tel acteur dans son giron plutôt que de le voir se développer comme concurrent. Deuxième 
La certification de contenus collaboratifs à l'agence photo Citizenside Entre procédures nouvelles et fondamentaux anciens

élément: l'Agence France-Presse bénéficie via Citizenside de l'apprentissage d'une expertise dans la vérification et la gestion de contenus issus d'Internet. De son côté, l'agence photo a pu assurément bénéficier grâce à son " partenariat » avec I'AFP d'une visibilité, renforcée par sa production sur de nombreux événements de portée nationale (premières images du trader de la Société générale, Jérôme Kerviel, en janvier 2008 lors de sa garde à vue à la brigade financière, après la perte par la banque de 5 milliards d'euros ; première vidéo, datée de décembre 2010, des insultes à caractère antisémite proférées par le styliste de la maison Dior John Galliano, lors de sa récidive quelques semaines plus tard en février 2011) ou de portée internationale (révoltes dans le monde arabe en 2011 ; accord avec le quotidien britannique The Guardian pour la couverture du Tour de France 2011).

Les exemples d'événements précédemment mentionnés sont les plus rémunérateurs pour Citizenside et même ceux ayant assuré sa survie économique. On peut saisir grâce à eux l'intérêt, pour les médias installés, de la production collaborative : Philippe Checinski (cofondateur de Citizenside) parle même d'images ayant contribué à "l'évangélisation de l'agence auprès des journalistes » (entretien avec l'auteur, 13/09/2011), dans la mesure où de tels scoops et la prise de participation de l'AFP ont permis non seulement de faire connaître Citizenside aux confrères professionnels, mais aussi de lui assurer une certaine crédibilité. Allons plus loin, par une mise en perspective de ce dispositif, telle que proposée par Francis Pisani et Dominique Piotet :

"L'application du crowdsourcing au marché de la photographie bénéficie de deux circonstances importantes: 1. En matière de photojournalisme, il est indispensable d'être sur place au moment où un événement a lieu. Or, si les journalistes ne sont pas toujours présents, les témoins équipés de mobiles ne manquent pas ; 2. Le travail collaboratif est généralement plus facile quand on peut juger sur pièce. C'est aussi le cas des photos (...) il faut fournir des preuves convaincantes qui "parlent d'elles-mêmes" » (Pisani et Piotet, 2008, p. 109).

On le comprend, si l'AFP s'est engagée dans la voie d'un partenariat avec la jeune agence photo, c'est à la fois que malgré son réseau elle ne peut prétendre à l'ubiquité totale (autrement dit, avoir un photographe " à chaque coin de rue ") et que Citizenside a su mettre au point des procédures garantissant la validité de ses contenus. 


\section{La "certification" des contenus UGC : techniques et processus de vérification}

L'agence Citizenside est confrontée à deux défis majeurs : stabiliser son modèle économique et renforcer la certification des images - 300 à 500 par jour, pour rappel - qu'elle propose en aval aux médias traditionnels (journaux, magazines, sites web, télévisions). Nous allons ici examiner le second défi, celui de la "certification", qui s'avère être la principale activité de la rédaction. Comment s'opère ladite vérification des contenus photo et vidéo ? Ces contenus issus d' " amateurs » impliquent-ils des opérations techniques spécifiques ? Où est-ce leur gestion qui s'avère nouvelle?

\subsection{Images d'actualité, actualité et fiabilité des images}

En premier lieu, il convient de préciser - ce que soulignent aussi les responsables économiques et éditoriaux de l'agence auprès desquels nous avons mené nos entretiens de recherche - que Citizenside n'occupe pas le même créneau que Fotolia, Flickr ou les réseaux sociaux (Facebook et YouTube en tête). Ces réseaux font circuler les contenus de toute nature, en particulier à caractère privé. Le matériau de Citizenside est, lui, labellisé "news ", autrement dit " actualité ». Que cela suggère-t-il ? Deux choses majeures. D'une part, les contenus reçus par la rédaction sont de nature plus sérieuse et plus réputée que les autres modèles précédemment cités; en ce sens, les griefs formulés vis-à-vis de l'appauvrissement de la culture et de l'information par le «culte de l'amateur » (Keen, 2008) ne peuvent pas ici être retenus. D'autre part, comme le rappellent là encore les membres de l'agence, l'extrême majorité des contributeurs sont le plus souvent "honnêtes ": ils cherchent essentiellement - plutôt qu'à la fausser, la manipuler ou la monnayer - à partager l'information (Hermida, 2011) de façon rapide mais sérieuse, et à cet égard ils sont enclins à le faire davantage par le biais d'images (des photos et, dans une moindre mesure, des vidéos) que par l'envoi de contenus textuels.

Parmi les stéréotypes attribués au journalisme participatif : des amateurs non qualifiés, à la fiabilité moindre que les professionnels reconnus et salariés de l'information. Une telle acception repose bien souvent sur la crainte des rédactions traditionnelles vis-à-vis de flux d'images qui circulent en nombre élevé, et sur la difficulté qu'elles pressentent dans le traitement de ces flux, que ce soit par manque de temps, de ressources financières et/ou techniques: en clair, l'intégration des usagers dans la production de l'information se révèle bien souvent, pour les journalistes, "contre-intuitive» (Domingo, 2008). Ajoutons 
La certification de contenus collaboratifs à l'agence photo Citizenside Entre procédures nouvelles et fondamentaux anciens

que la mauvaise réputation des images dites «amateurs " est renforcée par les quelques canulars dont ont été l'objet ces mêmes rédactions dans des périodes récentes. Or, cette acception de moindre fiabilité n'apparaît pas valide dans le cas de Citizenside. Ce, pour deux raisons. La première est que le site, bien qu'étant un outil en ligne et bien qu'alimenté par les photographes contributeurs, n'est pas produit et géré par ces derniers. II n'est pas un « wiki » pour lequel le mode de publication et de correction serait libre. II relève du "participatif encadré » (Rebillard, 2011, p. 32) : il est non seulement modéré, mais surtout contrôlé et administré par la rédaction de l'agence photo. $A$ fortiori, celle-ci est composée de journalistes professionnels (deux permanents et trois pigistes et/ou stagiaires, auxquels s'ajoutent une "community manager») qui, bien qu'assez jeunes, ont pour certains exercé auparavant dans plusieurs médias traditionnels tels la prestigieuse BBC, Radio Canada, l'hebdomadaire Le Nouvel Observateur, ou encore les gratuits Metro et 20 Minutes. La seconde raison conduisant à réfuter le terme d'" amateur", mais à préférer celui de «journalisme participatif » ou, plus encore pour notre part, de «journalisme collaboratif », est plus importante : les photos subissent plusieurs opérations de contrôle. Cela prend un temps certain mais c'est un indicateur du respect des standards qualitatifs du journalisme, en particulier ceux liés à la véracité de l'information, à l'investigation et au croisement des sources - ce qui renaît aujourd'hui sous la vulgate de « fact checking » ou « vérification des faits ».

\subsection{Vérification des images : métadonnées et géolocalisation}

La vérification proprement dite de l'authenticité des contenus - et des informations qui l'accompagnent - est fondée à la fois sur une maîtrise des normes techniques liées à ces mêmes contenus et sur l'échange fréquent avec les témoins d'actualité. En l'occurrence, le travail quotidien de la rédaction de Citizenside consiste à contrôler les métadonnées associées aux fichiers visuels ou audiovisuels chargés par les utilisateurs (dans la partie back-office du site, non visible), autrement dit les informations techniques contenues dans ces photographies ou vidéos. En la matière, la vérification initiale est globale et permet de procéder à deux repérages majeurs :

- 1. cerner la régularité et la fiabilité de l'auteur, les profils étant renseignés et complets, y compris avec coordonnées téléphoniques, et composés, comme dans le "social gaming", de grades (cf. Figure 1) ; 


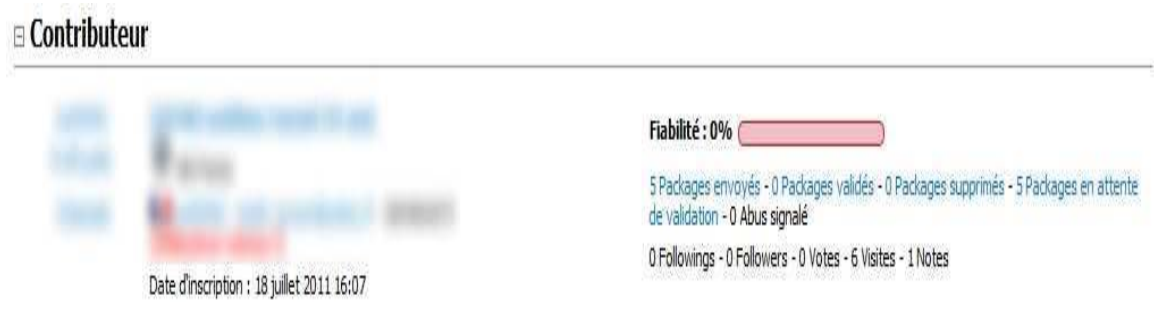

Figure 1: Cas d'un usager nouveau dont le profil alerte nécessairement (contrairement à un contributeur ancien et reconnu comme fiable).

- 2. examiner si les données habituelles apparaissent dans leur intégralité. En la matière, comme le souligne Nicolas Filio, ancien rédacteur en chef adjoint de la rédaction, devenu rédacteur en chef fin 2011 :

\begin{abstract}
"On pourrait se risquer à dire que la première information apportée par les métadonnées est... leur absence. Lorsque ces informations manquent, il est fort probable que l'image ne soit pas originale, qu'elle ait été simplement passée dans un logiciel qui écrase ces données ou qu'elle ait été prise sur un site web » (Filio, 2011, p. 76).
\end{abstract}

Nos entretiens et une démonstration technique qui nous a été faite à Citizenside nous ont permis de cerner trois catégories de données : les données primaires, les données EXIF et les données de géolocalisation. Les données primaires comprennent le nom de chaque fichier, sa taille, son extension, sa résolution et ses dimensions en pixels ; ces données sont celles qui alertent le plus aisément sur de possibles manipulations par l'usager, surtout si elles sont croisées avec celles des images de la même série. La deuxième catégorie est celle des données EXIF (eXtended Image File format) : hauteur et largeur de la photo, dates de prise de vue et de chargement sur le profil de l'usager, logiciel, balance des blancs, type d'appareil et modèle utilisé ; c'est cette seconde catégorie de données à laquelle l'actuel rédacteur en chef de l'agence fait référence quand il évoque l'«absence d'informations ", autrement dit des champs habituellement renseignés, mais qui apparaîtraient vides quand la photo chargée n'est pas issue d'une prise de vue dans les conditions classiques (cf. Figure 2). II est d'ailleurs à noter que pour renforcer ce dispositif, la rédaction demande aux auteurs de ne publier que des fichiers originaux, non retravaillés y compris concernant les contrastes. 


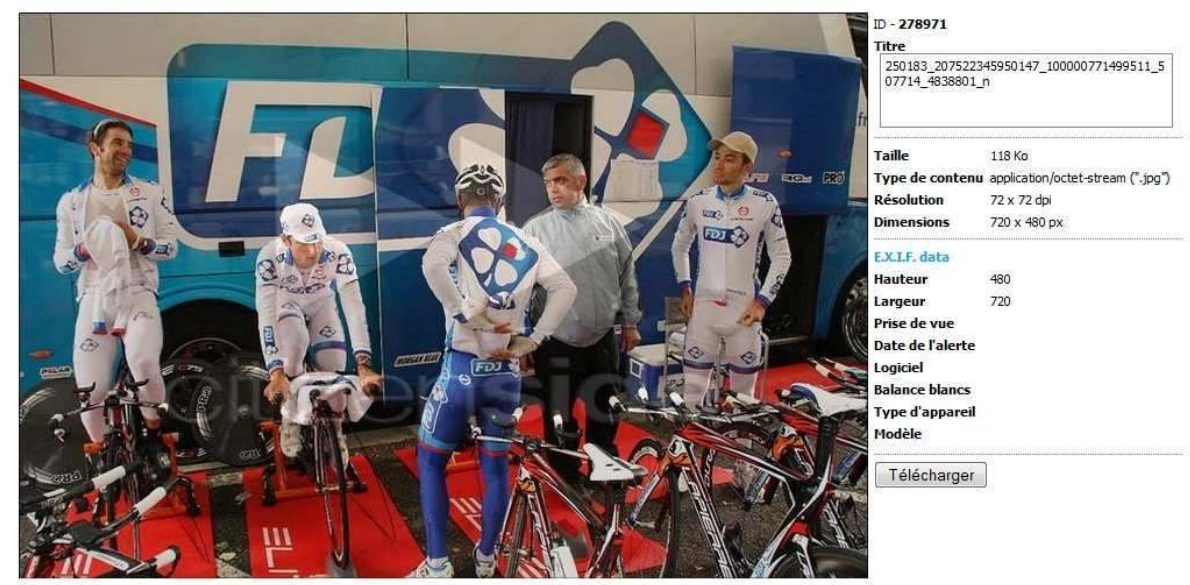

Figure 2 : Back-office du site Citizenside, présentant une photographie (piratée) et ses métadonnées associées (dont de nombreux vides)

La troisième catégorie, à savoir les données de géolocalisation (cf. Figure 3), permet enfin de détecter les éventuelles incohérences entre la résidence habituelle d'un membre et le lieu de l'événement pour lequel il a chargé des documents et surtout le lieu depuis lequel se fait ce chargement. 
Jérémie NICEY

Reçu le : 19 juillet 2011 17:27

Statistiques 0 Visites - 0 Votes - 0 Commentaires F Like $\$$ Tweet 0

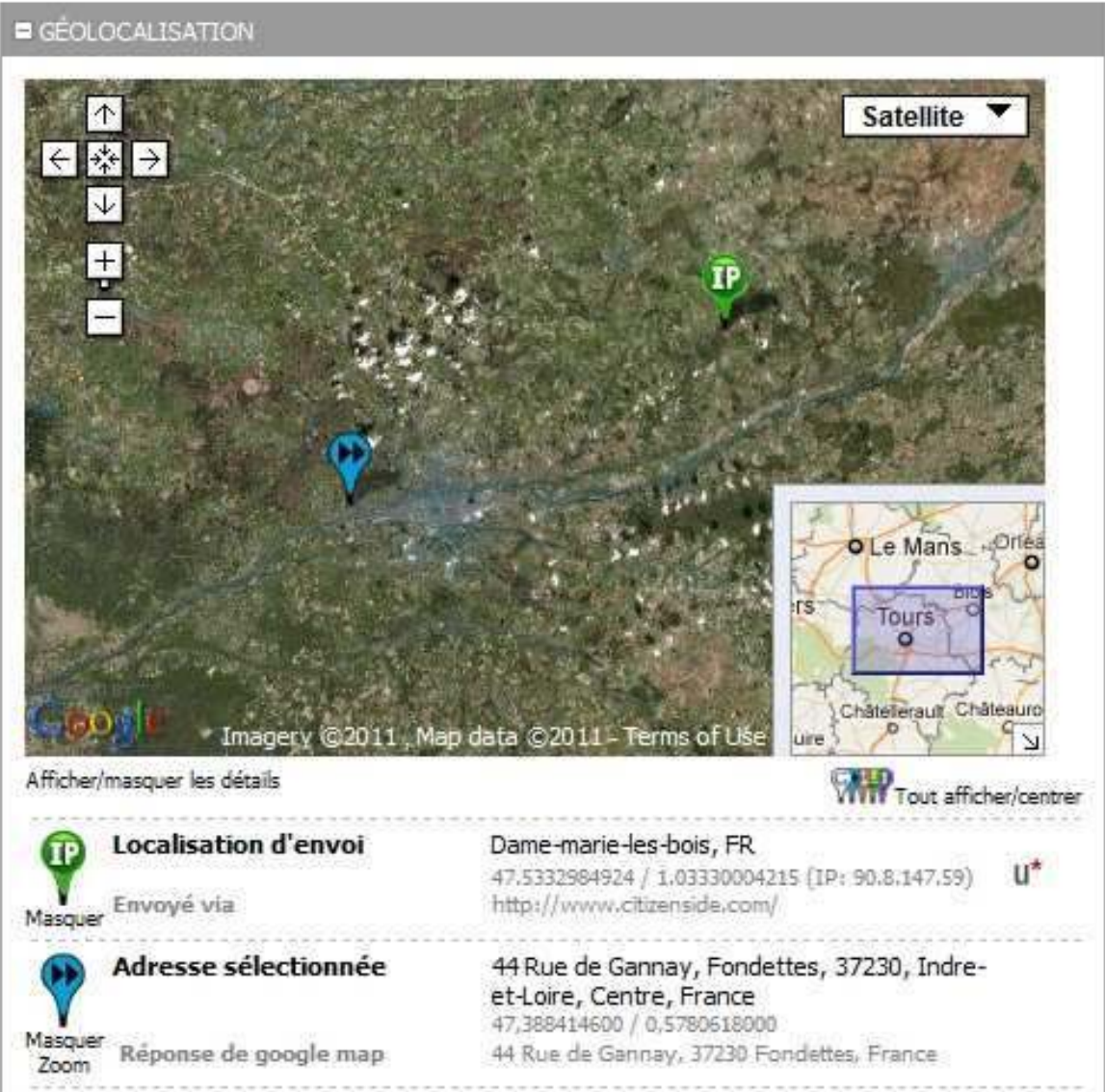

Figure 3 : Back-office du site Citizenside, avec localisation habituelle du contributeur et celle de son envoi d'images 
La certification de contenus collaboratifs à l'agence photo Citizenside Entre procédures nouvelles et fondamentaux anciens

Ce dernier élément, issu de développements technologiques récents, est très utilisé par Citizenside (notamment pour les "alertes", cf. infra), mais encore peu par les rédactions traditionnelles.

\subsection{Investigation, traçage web et interactivité}

Pendant les vérifications, les images chargées par les utilisateurs sont labellisées «en attente de validation» dans la partie non visible du site, autrement dit le «back-office» (à noter, pour cette raison, que Citizenside n'accepte pas les envois par e-mail). Le délai avant publication peut durer si des doutes sont manifestes ; dans ce type de situation, la rédaction procède alors à une deuxième étape d'investigation. Elle va chercher la trace du contenu suspect sur le web pour en retrouver, grâce à la recherche inversée, l'original, que ce soit sur les réseaux sociaux (Facebook, Flickr, YouTube, etc.) ou sur les moteurs de recherche et les sites de photos en ligne (Google, Tineye, Fotolia, etc.). Soulignons que cette même démarche - limiter les canulars et le plagiat à l'aide d'outils hautement technologiques - est au fondement de deux innovations actuelles à l'Agence France-Presse : d'une part le développement, en interne et dont l'agence a pour l'instant l'exclusivité, d'un logiciel (Tungstène) capable à l'aide d'un système de filtres de repérer les retouches de photos ; d'autre part, entre 2009 et 2012, la participation (conjointement avec Citizenside) à un projet de recherche («GLOCAL »), financé par la Commission européenne et associant onze partenaires, qui vise à « fournir aux amateurs un environnement interactif pour décrire et catégoriser les événements dont ils sont témoins, en faisant le pont entre leur représentation locale et celle, globale, des médias amenés à les publier » (Teyssou, 2010, p. 5, notre traduction).

Les vérifications techniques décrites plus haut se doublent d'un contact - par mail ou par téléphone - avec l'expéditeur des images pour s'assurer de multiples points : d'abord, qu'il en est bien l'auteur ; que son récit corrobore celui d'autres journalistes ou témoins du même événement; que les aspects liés aux lieux s'avèrent cohérents; etc. Ce travail est assuré par le journaliste chargé du dossier, ou par le rédacteur en chef lui-même, ou par la " community manager " (animatrice de communauté). Cette dernière occupe d'ailleurs une position-clé de plus en plus valorisée dans la majorité des rédactions, y compris traditionnelles, devant l'ampleur prise par les réseaux sociaux. Le principe est de rester au contact des «membres » en répondant à leurs questions, en cernant leurs profils le plus finement possible pour maîtriser et anticiper leurs productions, voire les susciter. Ce point précis s'avère même stratégique : la rédaction lance régulièrement des "alertes" à sa communauté (en s'aidant 
entre autres des outils de géolocalisation) pour lui demander de produire des images de certains événements. Last but not least, le lien tissé avec les contributeurs passe même parfois par des conseils pour améliorer la qualité technique de leurs prises de vue, et surtout par la pédagogie de la contextualisation de leurs images : en clair, la rédaction demande aux membres postant des photos de décrire l'événement dont ils ont été témoins, dans un format qui respecte les règles du récit journalistique, en particulier celle des $5 \mathrm{~W}$. Dans l'ensemble, et comme le précise Nicolas Filio, le rédacteur en chef (entretien avec l'auteur le 21/07/2011 - il était à l'époque rédacteur en chef adjoint), ces procédés font que les échanges avec la communauté de Citizenside se révèlent extrêmement plus poussés que les rédactions classiques avec leurs publics. Surtout, l'agence Citizenside met en avant une terminologie singulière: elle porte l'ambition de passer du modèle UGC (pour User Generated Content) à celui d' «UCC» (pour "User Certified Content»). On saisit là un gage pour elle de sérieux et de reconnaissance par les pairs (Bruns, 2010).

\section{Un « renouvellement » du journalisme de qualité ?}

Les processus de certification des agences de contenus dits " amateurs " semblent dessiner un journalisme contributif de qualité (Wittke et Hanekop, 2011). Ces processus se font-ils sur la base de règles nouvelles, spécifiques à ces nouveaux acteurs ? Les procédés de vérification technique mis en œuvre ne vont-ils pas, même, au-delà des pratiques traditionnelles, parfois défaillantes (Heinderyckx, 2003) ? Le sujet est décisif, dans la mesure où les nouveaux acteurs bousculeraient alors le paysage non seulement dans son offre, mais dans ses pratiques et ses routines professionnelles.

\subsection{Le réflexe de vérification technique : généralisable}

Première appréciation : si les opérations de vérification technique explicitées plus haut suggèrent de réelles compétences pour l'investigation, il convient de penser qu'elles sont en réalité généralisables (après une phase d'apprentissage) aux rédactions des médias mainstream. C'est d'ailleurs, en substance, ce que propose l'agence pour compléter ses finances: elle assure en effet des formations d'investigation web sur les images d'actualités, y compris auprès des agenciers AFP. 
La certification de contenus collaboratifs à l'agence photo Citizenside Entre procédures nouvelles et fondamentaux anciens

Deuxième point, en réalité corollaire : la vérification des photos et vidéos d'information est ce qui fait aujourd'hui la singularité de l'agence Citizenside, voire son expertise ; mais il convient de rappeler que la vérification est avant tout... un des fondamentaux supposés de la pratique journalistique, un des critères essentiels de qualité. Or, dans les rédactions traditionnelles, le croisement de données techniques pour assurer la fiabilité des contenus n'est pas la norme. Loin de là : à l'heure de l'abondance et de la rapidité accrue de la production des news, les cas de défaillances journalistiques vis-à-vis de canulars se multiplient - en témoigne la circulation, y compris sur des médias "reconnus ", de la fausse photo de Ben Laden le 2 mai 2011 dans les heures suivant sa mort, ou encore, pour l'AFP elle-même et lors du séisme à Haïti en janvier 2010, celle d'une photographie en réalité piratée, commercialisée et reprise en une par de nombreuses publications print, dont Libération (Palmer, 2011, pp. 395-397).

En ce sens, il est intéressant d'interroger les discours, actuellement assez répandus, cherchant à établir que les acteurs des médias participatifs seraient en quelque sorte des journalistes "d'une nouvelle espèce ". Si l'on s'arrête au caractère technique et principal de leur tâche, cela peut sembler valide ; mais, eu égard à la définition du journalisme, cela est fortement à nuancer, comme en atteste le positionnement de l'actuel rédacteur en chef de Citizenside :

"La technique ne doit pas faire perdre de vue les éléments humains de la vérification : I'histoire tient-elle debout? L'image est-elle réaliste ? Ce sont des questions qu'il faut garder à l'esprit. Lorsqu'on est confronté à des images, la première chose à faire, c'est logique, est d'ouvrir l'œil. Étudier l'arrière-plan d'une photo ou d'une vidéo peut par exemple permettre de savoir où la scène se déroule. II faut également savoir saisir les détails "qui ne collent pas ". Comme ces manifestants présentés par France 2 comme étant iraniens. C'était en décembre 2009. On les voyait lutter en t-shirt contre des policiers casqués. Pas vraiment conforme aux conditions climatiques à Téhéran à l'approche de Noël. Et pour cause : ces images avaient en réalité été prises au Honduras en juin » (Filio, 2011, p. 77). 
Jérémie NICEY

\subsection{Les images d'information : besoin de recul}

Le point de vue que nous venons de citer, que l'auteur qualifie lui-même de "logique ", suggère à la fois observation, positionnement critique et connaissances - que ces dernières soient spécialisées ou non. Pour le dire autrement: du recul. Recul que bien des professionnels actuels des médias disent s'autoriser de moins en moins, en raison des flux et de la pression $d u$ temps; nous retrouvons pourtant là deux contraintes qui, elles, ne sont pas nouvelles, en particulier pour les agences d'information (Palmer et Nicey, 2011) dont c'est le principe voire le modèle. En outre, l'extrait précité vient confirmer le glissement du pouvoir des acteurs médiatiques vers le "gatewatching (Bruns, 2005). Le rôle traditionnel d'« éclusier » (ou "gatekeeper», White, 1950; Shoemaker et al., 2001) dont ils avaient l'apanage jusqu'à l'apparition de réelles plateformes collaboratives peut en effet expliquer le sentiment de menace et de réticence qui est bien souvent encore le leur vis-à-vis de l'information en ligne. Pourtant, dans les faits, les limites se situent davantage du côté des agences participatives. Ainsi les écueils de la rédaction de Citizenside sont à la fois de ne pas être assez fournie et de ne pas pouvoir opérer $24 \mathrm{~h} / 24$, alors même que l'information internationale, par définition ancrée sur des fuseaux horaires multiples, le demanderait; a contrario, une agence telle que l'AFP travaille évidemment jour et nuit, et dispose de quelque 2900 collaborateurs de 80 nationalités, présents dans 165 pays.

\subsection{Les contributions : entre spontanéité et sollicitations}

Qu'en est-il des contributeurs ? À Citizenside, ces "générateurs de contenus " (francisation du terme "UGC ») sont plus de 70 000, mais 10000 seraient réellement actifs - données à mettre en perspective avec le million de contributeurs à iReport, le site participatif de CNN. Leur rôle diffère-t-il tant des photographes exerçant pour les médias établis ? À cela, on osera répondre par la négative. Pour deux raisons.

D'une part, leur statut s'apparente à celui des pigistes dans les médias traditionnels ou, à tout le moins, à celui des freelance : ils proposent des images ou peuvent capturer un événement sur demande (cf. supra le système d'«alertes"), et ils sont rémunérés en proportion de la publication de leurs contenus - même si dans le cas des «membres » de Citizenside l'argent ne constitue pas leur première motivation (Aubert, 2012, pp. 4-5). La logique va même plus loin pour certains d'entre eux: Citizenside compte en effet une dizaine d'«usagers certifiés ", autrement dit d'une extrême fiabilité, qui bénéficient de la possibilité de publication autonome (avec la mention «Info 
La certification de contenus collaboratifs à l'agence photo Citizenside Entre procédures nouvelles et fondamentaux anciens

brute ») ; nous avancerons que, pour l'agence, cela est un moyen externe de consolider la rédaction et d'accroître sa réactivité. Comme pour les agences traditionnelles, les photographies sont issues d'un processus qui combine spontanéité (de leurs auteurs) et sollicitations (de la part de la newsroom).

D'autre part, grâce à la démocratisation des outils de capture qu'il s'agisse des appareils photo ou des smartphones, de plus en plus performants, mais à des coûts restant pourtant accessibles, les contributeurs atteignent de plus en plus des niveaux de qualité de production proches des standards et normes des professionnels. C'est en ce sens qu'il apparaît de moins en moins pertinent de les qualifier d'« amateurs "; il convient davantage de les considérer comme des "semi-professionnels ", voire comme des photojournalistes à part entière... mais entièrement à part. Sans aller jusqu'à considérer que ces citoyens "sont " ou « deviennent » le média lui-même (Bowman et Willis, 2003 ; Gillmor, 2004), il reste donc frappant que les organisations médiatiques traditionnelles n'aient à ce jour été que peu nombreuses à en déceler le potentiel.

\section{Pour conclure... provisoirement : une démarche pas si nouvelle et un paradoxe}

Les procédures des médias participatifs se révèlent pertinentes pour l'analyse, dans la mesure où elles renouvellent les pratiques journalistiques classiques en même temps qu'elles en réactivent les prérequis - tout du moins tels qu'ils sont enseignés aux jeunes intégrant la profession. À l'agence photo Citizenside, une image proposée par un contributeur lambda n'échappe pas au filtre de la vérification, constituée de trois étapes principales : observation des métadonnées associées aux images reçues; recherches/croisements avec les contenus déjà existants en ligne ; contact des expéditeurs des images/activation de la communauté. Ces procédures, si elles sont destinées à assurer la fiabilité des contenus proposés en bout de chaîne aux clients médias, peuvent certes sembler nouvelles dans leur forme, mais elles ne le sont pas dans les fondamentaux journalistiques auxquels elles font appel. Dans la mesure où le terme «nouveau » ne prend de sens qu'en référence à ce qui le précède et est " ancien », il convient de souligner qu'à Citizenside les outils de vérification sont nouveaux, mais que les préceptes décrits ne le sont pas. Dès lors apparaît un paradoxe: Citizenside semble innover en remettant au goût du jour des pratiques anciennes, dont les confrères des médias installés s'étaient éloignés depuis plusieurs décennies. 
En outre, à la lumière de nos observations et entretiens initiaux à Citizenside, et de nos recherches sur la production même d'un de ses actionnaires, l'AFP, nous conclurons provisoirement que le partenariat entre acteurs établis et naissants paraît plus bénéfique que l'affrontement. Les agences telles que Citizenside et ses équivalents à l'étranger, en apportant structuration et rationalité dans la circulation des images dites "amateurs", y invitent fortement. II leur reste pourtant à gagner en réputation : par leur position et leur poids actuels, elles ne sont pas à même d'influencer le paysage médiatique et ses mécanismes pervers, très ancrés. Ce qu'elles feront peut-être, en s'affirmant...

Ce dernier point doit cependant conduire à la prudence : rien ne dit qu'en gagnant en notoriété et en légitimité, les nouveaux acteurs - du journalisme participatif, mais aussi plus globalement de toute nature - ne seront pas soumis à la pression desdits mécanismes pervers du secteur. Ceux-ci sont en effet à même d'altérer les pratiques observées, que l'on peut considérer dans une certaine mesure comme actuellement positives en matière de vérification technique, mais qui nécessitent de faire leur preuve dans la durée. En outre, l'étude de l'utilisation de telles images par les rédactions demanderait à être approfondie à la fois pour cerner ce qu'elle induit pour l'écosystème professionnel de collecte et d'illustration des événements médiatiques - en clair, la transformation de cet écosystème et la concurrence subie par les photojournalistes - et pour scruter les pratiques et motivations des contributeurs eux-mêmes. In fine, les pratiques nouvelles et les dispositifs nouveaux initiés par des petites entités ont ceci de particulièrement intéressant qu'elles constituent un défi à la fois pour le secteur dans lequel ces entités opèrent, et pour ces entités elles-mêmes.

\section{Références bibliographiques}

ALLAN S. et E. THORSEN (dir.), 2009, Citizen journalism : global perspectives, New York, Peter Lang.

AUBERT A., 2012, "La commercialisation des images amateur dans le domaine informationnel: de nouveaux acteurs dans la production de l'information ", dans Les Enjeux de l'information et de la communication, [http://w3.u-grenoble3.fr/les enjeux/2011-supplement/Aubert/index.html], dernière consultation le 2 février 2012.

BOUQUILLION P. et J. MATTHEWS, 2010, Le Web collaboratif. Mutations des industries de la culture et de la communication, Grenoble, Presses Universitaires de Grenoble. 
La certification de contenus collaboratifs à l'agence photo Citizenside Entre procédures nouvelles et fondamentaux anciens

BOWMAN S. et C. WILLIS, 2003, «We Media : How audiences are shaping the future of news and information». Report for The Media Center at The American Press Institute, [http://www.hypergene.net/wemedia/weblog.php], dernière consultation le 5 juillet 2011.

BOYD-BARRETT O. (dir.), 2010, News Agencies in the Turbulent Era of the Internet, Barcelona/Generalitat de Catalunya, Lexikon.

BRUNS, A., 2005, Gatewatching: collaborative online news production, New York, Peter Lang.

BRUNS, A., 2010, "News produsage in a Pro-Am Mediasphere", dans G. MEIKLE et G. REDDEN (dir.), News Online: Transformations and Continuities, London, Palgrave Macmillan.

DOMINGO D., 2008, «Interactivity in the daily routines of online newsrooms : dealing with an uncomfortable myth ", Journal of Computer-Mediated Communication, vol. 3, $\mathrm{n}^{\circ} 3$ (avril), pp. 680-704.

FILIO N., 2011, "Médias du 21e siècle: passer du public passif aux contributeurs actifs ", dans B. DELFORCE et Y. ESTIENNE (dir.), « Le journalisme numérique: formes, formats, frontières ", Les Cahiers du journalisme, $n^{\circ} 22 / 23$, pp. 74-83.

GILLMOR D., 2004, We the Media. Grassroots Journalism by the People, for the People, Sebastopol, O'Reilly.

HEINDERYCKX F., 2003, La malinformation, Bruxelles, Liber.

HERMIDA A., 2011, "Mechanisms of Participation: How Audience Options Shape the Conversation », dans J. SINGER et al., Participatory journalism : Guarding open gates at online newspapers, Malden, MA : Wiley-Blackwell, pp. 13-33.

JENKINS H., 2006, Convergence Culture: Where Old and New Media Collide, New York, New York University Press.

KEEN A., 2008, Le Culte de l'amateur, Paris, Scali (version française).

LASICA J., 2003, "What is Participatory Journalism ", Online Journalism Review, [http://www.ojr.org/ojr/workplace/1060217106.php], dernière consultation le 21 avril 2011.

MILLERAND F., S. PROULX et J. RUEFF, 2010, Web social, Mutation de la communication, Québec, Presses de l'université du Québec. 
Jérémie NICEY

NICEY, J., 2008, « Le tsunami asiatique de 2004 vu par la télévision suédoise : les retombées locales d'une catastrophe lointaine ", dans A. AUBERT et M. PALMER (dir.), L'information mondialisée, Paris, L'Harmattan, pp. 179185.

PALMER, M. et BOYD-BARRETT, 1981, Le Trafic des nouvelles, Paris, Alain Moreau.

PALMER, M., 2011, Homo informans. L'urgence des news au fil des millénaires, Paris, L'Amandier.

PALMER, M. et J. NICEY, 2011, Lexique subjectif de l'homme informant, Paris, L'Amandier.

PAULUSSEN, S., HEINONEN, A., DOMINGO, D., et T. QUANDT, 2007, «Doing It Together: citizen participation in the professional news making process », Observatorio (OBS) Journal, vol. 1, $\mathrm{n}^{\circ} 3$, pp. 131-154, [http://obs.obercom.pt/index.php/obs/article/view/148/107], dernière consultation le 10 septembre 2011.

PISANI, F. et D. PIOTET, 2008, Comment le web change le monde. L'alchimie des multitudes, Paris, Pearson.

REBILLARD, F., 2007, Le Web 2.0 en perspective. Une analyse socioéconomique de l'internet, Paris, L'Harmattan.

REBILLARD, F., 2011, “ Création, contribution, recommandation : les strates du journalisme participatif », dans B. DELFORCE et Y. ESTIENNE (dir.), « Le journalisme numérique: formes, formats, frontières », Les Cahiers $d u$ journalisme, $\mathrm{n}^{\circ} 22 / 23$, pp. 28-41.

ROSEN, J., 2011, “ From "write us a post" to "fill out this form :" Progress in ProAm journalism », Speech to Personal Democracy Forum, June 7, [http://pressthink.org/2011/06/from-write-us-a-post-to-fill-out-this-formprogress-in-pro-am-journalism/].

SHOEMAKER P., M. EICHHOLZ, E. KIM et B. WRIGLEY, 2001, "Individual and routine forces in gatekeeping". Journalism \& Mass Communication Quarterly, $n^{\circ} 78$, pp. 233-246.

SINGER J. B., D. DOMINGO, A. HEINONEN, A. HERMIDA, S. PAULUSSEN, T. QUANDT, Z. REICH et M. VUJNOVIC, 2011, Participatory journalism : Guarding open gates at online newspapers, Malden, MA : Wiley-Blackwell. 
La certification de contenus collaboratifs à l'agence photo Citizenside Entre procédures nouvelles et fondamentaux anciens

TEYSSOU D., 2010, «GLOCAL: Pro-Am collaboration in the news production ", communication à la $6^{\text {th }}$ Hellenic conference on artificial intelligence, "Events 2010. Recognizing and tracking events on the web and in real life ", Athènes, mai.

WHITE D. M., 1950, "The "gate-keeper": A case study in the selection of news ", Journalism Quarterly, $n^{\circ}$ 27, pp. 383-390.

WITTKE V. et H. HANEKOP (dir.), 2011, New Forms of Collaborative Innovation and Production on the Internet: An Interdisciplinary Perspective, Universitätsverlag Göttingen, [http://www.univerlag.unigoettingen.de/content/list.php?notback=1\&details=isbn-978-3-86395-020-0], dernière consultation le 5 juillet 2011. 